\title{
Prise en charge des patients après un pontage aortocoronarien : guide pour les professionnels en soins primaires
}

\author{
Dominique de Waard MD, Andrew Fagan MD, Christo Minnaar MD, David Horne MD
}

- Citation : CMAJ 2021 May 10;193:E689-94. doi : 10.1503/cmaj.191108-f

Voir la version anglaise de l'article ici : www.cmaj.ca/lookup/doi/10.1503/cmaj.191108

E nviron 15000 personnes subissent un pontage aortocoronarien (PAC) chaque année au Canada ${ }^{1}$. Selon le Rapport sur les indicateurs de la qualité des soins cardiaques de 2019 de l'Institut canadien d'information sur la santé, 9,4\% de ces patients sont réhospitalisés d'urgence dans les 30 jours suivant l'opération ${ }^{1}$. Étant donné le vieillissement de la population canadienne, la chirurgie cardiaque est offerte à des patients plus âgés, plus malades et plus fragiles, qui présentent un risque plus élevé de réadmission après leur intervention chirurgicale ${ }^{2}$. Une étude rétrospective a montré un taux de réadmission après 30 jours de $15 \%-20 \%$ chez les patients de plus de 80 ans ayant subi une chirurgie cardiaque dans un centre donné2. Par ailleurs, l'absence de consultations externes auprès d'un professionnel en soins primaires dans les 30 jours suivant le congé était associée à un risque d'atteinte à la survie fonctionnelle à 1 an 5 fois plus élevé (rapport de risque $5,18, p<0,01)^{2}$. Ces résultats soulignent le rôle important que jouent les professionnels en soins primaires dans la prise en charge des patients à la suite d'un pontage aortocoronarien.

La plupart des pontages sont réalisés en conjonction avec une dérivation cardiopulmonaire qui affecte tout particulièrement la physiologie des patients et mène à des complications postopératoires spécifiques chez certains. La dérivation cardiopulmonaire facilite l'obtention d'un champ opératoire immobile et d'une intervention non hémorragique, et elle permet de maintenir l'oxygénation du sang et la perfusion des tissus en faisant circuler le sang veineux du patient dans un oxygénateur mécanique. Cependant, le contact du sang avec les surfaces non endothélialisées du circuit extracorporel déclenche un intense syndrome de réponse inflammatoire systémique et mène à l'activation du système de coagulation. Bien que l'héparine soit systématiquement administrée pour atténuer le risque de caillots, le syndrome mène aussi à d'importants transferts liquidiens, à la dysrégulation des lits de capillaires et de ce fait, à l'hypoperfusion des tissus, malgré la rétention des liquides ${ }^{3}$. L'hypoperfusion associée au syndrome de réponse inflammatoire systémique, combinée à des micro-emboles, peut entraîner des dommages systémiques aux organes et une morbidité postopératoire. Les patients peuvent présenter des douleurs

\section{POINTS CLÉS}

- Des données récentes révèlent que près de $10 \%$ des patients qui subissent un pontage aortocoronarien sont hospitalisés de nouveau dans les 30 jours suivant l'intervention chirurgicale.

- Les patients qui n'ont pas de suivi en soins primaires ont montré les pires résultats à long terme.

- Plusieurs complications qui se manifestent après un pontage aortocoronarien sont liées à une réponse inflammatoire systémique associée à la circulation extracorporelle.

- Les professionnels en soins primaires jouent un rôle déterminant dans la détection des complications du pontage aortocoronarien et dans la prestation des soins postopératoires.

thoraciques, un essoufflement ou un dérèglement de la tension artérielle dans les semaines suivant l'intervention chirurgicale en raison du syndrome de réponse inflammatoire systémique.

Nous discutons de la prise en charge des syndromes cliniques postopératoires et des complications précoces ou tardives potentielles que les professionnels en soins primaires peuvent observer. Sont aussi soulignées d'importantes considérations en lien avec la médication et les activités, sur la base des pratiques exemplaires et des données probantes les plus récentes (encadré 1). Il est important de noter qu'un pontage aortocoronarien réalisé sans dérivation cardiopulmonaire (sans machine cœur-poumon) demeure une intervention chirurgicale majeure et que les patients peuvent présenter plusieurs des complications postopératoires abordées dans la présente revue.

\section{Quelles sont les causes des douleurs thoraciques après une chirurgie cardiaque?}

Il est important de prendre en considération les 3 《I I (infection, inflammation et ischémie) lorsqu'on considère les grandes causes de la douleur thoracique après une chirurgie cardiaque. La douleur est souvent liée au traumatisme mécanique de la sternotomie, auquel cas elle est localisée et exacerbée par le mouvement. 
Encadré 1 : Données probantes employées dans la présente revue

La recherche d'études et de lignes directrices en lien avec la chirurgie cardiaque et la prise en charge postopératoire a été réalisée à l'aide de Google Scholar, PubMed, la Société canadienne de cardiologie, l'American College of Cardiology/American Heart Association et la Société européenne de cardiologie/European Association for CardioThoracic Surgery. Les termes de recherche comprenaient « cardiac surgery », " postoperative management », « coronary artery bypass grafting » et des termes en lien avec les complications (p. ex., « atrial fibrillation », « chest pain »). Les lignes directrices, les revues systématiques, les essais cliniques randomisés et contrôlés, les études de cohorte et les études de cas les plus récents et les plus solides ont été employés pour éclairer la revue. Afin de décrire certaines pratiques et physiopathologies courantes, des manuels de chirurgie cardiaque pertinents ont aussi été consultés.

Un clic palpable suggère une instabilité médiastinale qui devrait être examinée rapidement par le chirurgien cardiaque. La douleur peut être associée à une infection de la plaie, laquelle peut aussi entraîner des douleurs localisées en plus des symptômes caractéristiques d'infection. Des lésions nerveuses survenues lors du prélèvement de l'artère thoracique interne pour la greffe pourraient entraîner une hypoesthésie ou hyperesthésie parasternale neuropathique. La péricardite (douleur inflammatoire) et l'ischémie myocardique sont étudiées plus en détail ci-dessous.

\section{Péricardite}

Les patients atteints de péricardite signalent souvent une douleur vague dans la partie antérieure du thorax. Elle peut être associée à un frottement péricardique audible à l'examen physique accompagné d'un sus-décalage diffus du segment ST ou d'un sousdécalage diffus du segment PR à l'électrocardiogramme (ECG).

Le syndrome postcommissurotomie est une péricardite immune qui peut se manifester de plusieurs semaines à plusieurs mois après une chirurgie cardiaque ${ }^{4,5}$. Selon les lignes directrices sur le diagnostic et la prise en charge des maladies péricardiques (Guidelines for the Diagnosis and Management of Pericardial Diseases) de 2015 de la Société européenne de cardiologie, ses caractéristiques cliniques comprennent une fièvre inexpliquée, des frottements péricardiques ou pleuraux, un épanchement pleural ou péricardique nouveau ou s'aggravant (bien que cet élément ne soit pas présent dans tous les cas) ainsi que des douleurs thoraciques péricardiques ou pleurétiques $^{6}$. Ces lignes directrices recommandent l'obtention rapide d'un électrocardiogramme à la suite du diagnostic afin de déterminer la présence d'un épanchement péricardique qui nécessiterait une série de séances d'imagerie de suivi ${ }^{6}$. Les traitements comprennent l'administration d'anti-inflammatoires non stéroïdiens (AINS), de colchicine et de stéroïdes, seuls ou en combinaison, selon les exigences du scénario clinique ${ }^{6,7}$. Le chirurgien cardiaque principal devrait être tenu informé puisqu'un drainage de l'épanchement pourrait être nécessaire.

La tamponnade cardiaque postopératoire est une complication rare du syndrome postcommissurotomie qui se manifeste généralement de façon précoce $(<7$ jours après la chirurgie), mais qui peut aussi se produire de façon tardive ( $>7$ jours) chez
$1 \%-12,6 \%$ des patients ${ }^{8}$. Elle s'accompagne de la triade de Beck (sons du cœur assourdis, distension de la veine jugulaire et hypotension), d'une tachycardie sinusale, d'un pouls paradoxal, d'une alternance électrique sur l'électrocardiogramme et d'un élargissement de la silhouette cardiaque à la radiographie thoracique $^{9}$. La tamponnade cardiaque tardive est associée à un taux de mortalité élevé et nécessite le transfert du patient au service des urgences et une intervention urgente ${ }^{10}$.

\section{Infarctus du myocarde après un pontage aortocoronarien}

L'occlusion ou la sténose des artères greffées ou d'artères coronaires non pontées (en raison de la progression de la maladie ou d'artères coronaires gravement atteintes étant trop petites ou pour lesquelles la greffe n'est pas possible) peuvent entraîner une douleur thoracique ischémique à la suite d'un pontage aortocoronarien. Cette douleur peut être typique ou atypique. L'ischémie peut aussi être sans douleur, ce qui est fréquemment observé chez les patients atteints de diabète avant l'intervention chirurgicale ${ }^{11}$, mais parfois aussi chez les patients qui ne sont pas diabétiques en raison d'une perturbation du plexus cardiaque autonome pendant la chirurgie.

L'infarctus du myocarde périopératoire précoce se produit après un pontage aortocoronarien dans 0,6\%-19\% des cas, selon le critère sérologique employé ${ }^{12}$. L'essai SYNTAX (Synergy Between Percutaneous Coronary Intervention with Taxus and Cardiac Surgery), qui compare l'intervention coronarienne percutanée et le pontage aortocoronarien pour le traitement des patients atteints d'une coronaropathie de l'artère coronaire gauche, a révélé un taux d'infarctus du myocarde tardif de 3,8\% (cote SYNTAX $\leq 32$ ) et de $6,1 \%$ (cote SYNTAX $\geq 33$ ) 5 ans après le PAC ${ }^{13}$. Une revue systématique Cochrane réalisée en 2016 a estimé que le taux d'occlusion du greffon après un pontage aortocoronarien est d'environ $2 \%-5 \%$ par année ${ }^{14}$. Afin de prévenir l'occlusion du greffon, la mise à jour de 2012 de la ligne directrice sur l'utilisation du traitement antiplaquettaire de la Société canadienne de cardiologie recommande l'emploi de ce traitement en conjonction avec la prise d'acide acétylsalicylique et de clopidogrel ou de ticagrélor pendant une année après un pontage aortocoronarien dû à un syndrome coronarien aigu. Cette recommandation est demeurée inchangée lors de la mise à jour de $2018^{15}$.

Comme il n'existe pas de lignes directrices précises décrivant la prise en charge de l'angor stable ou du syndrome coronarien aigu après un pontage aortocoronarien, la douleur thoracique ischémique récurrente après un PAC est diagnostiquée et prise en charge de façon comparable à la première manifestation de cardiopathie ischémique précédant le pontage. Tout patient qui manifeste des symptômes de syndrome coronarien aigu devrait être rapidement pris en charge par le service des urgences le plus près. La prise en charge médicale de l'angor stable devrait comprendre un contrôle des facteurs de risque cardiaques comme l'hypertension, le diabète, l'obésité, la dyslipidémie et le tabagisme, tel que proposé dans l'énoncé scientifique de 2015 sur la prévention secondaire après un pontage aortocoronarien de l'American Heart Association ${ }^{16}$. Les patients ayant un angor stable devraient être dirigés vers leur cardiologue. 


\section{Qu'est-ce qui cause l'essoufflement après une chirurgie cardiaque?}

Dans la période suivant immédiatement l'intervention, la plupart des patients présentent de la dyspnée, accompagnée ou non d'hypoxie, causée par la respiration fragmentée en raison de la douleur, d'atélectasie ou des deux. Cet état est pris en charge de façon énergique au moyen d'analgésiques, d'une mobilisation précoce, d'une spirométrie intensive et de physiothérapie ${ }^{17}$. Une nouvelle dyspnée peut se manifester après que les patients ont obtenu leur congé; les causes importantes qui devraient être exclues sont (de la plus probable à la moins probable) l'épanchement pleural, l'atélectasie, l'insuffisance cardiaque congestive, la pneumonie et l'embolie pulmonaire.

\section{Épanchement pleural}

En raison des facteurs préopératoires présentés plus haut, l'administration d'une quantité substantielle de liquides et de vasopresseurs est généralement nécessaire afin de maintenir une perfusion systémique postopératoire adéquate, ce qui entraîne un transfert liquidien du compartiment intravasculaire au compartiment interstitiel (troisième espace). À mesure que les patients se stabilisent, le passage des liquides de l'espace interstitiel vers l'espace intravasculaire et le traitement diurétique contribuent au retour à l'euvolémie, qui se poursuit souvent après le congé. Il n'est pas rare qu'au congé, les patients présentent de petits épanchements pleuraux qui s'améliorent de façon spontanée avec la résolution du syndrome de réponse inflammatoire systémique et la poursuite du traitement diurétique. Cependant, de nouveaux épanchements pleuraux ou une nouvelle accumulation de liquides peuvent se produire, le plus souvent causés par une surcharge liquidienne (p. ex., due à une diurèse inadéquate ou à une insuffisance cardiaque congestive). Les causes les moins fréquentes comprennent la sérite, les saignements (généralement chez les patients sous anticoagulothérapie) et, rarement, un chylothorax. La cause sous-jacente doit être établie et traitée, par exemple par la restriction de l'apport oral et l'augmentation de la diurèse dans les cas de rétention liquidienne, ou un traitement anti-inflammatoire en cas de sérite. Une discussion avec le chirurgien cardiaque du patient facilitera une prise en charge appropriée si un traitement effractif est requis.

\section{Atélectasie}

Si les patients demeurent mobiles après leur congé, les récidives d'atélectasie sont rares. Cependant, s'ils sont sédentaires ou s'ils ressentent une douleur thoracique qui mène à une hypoventilation, ils peuvent présenter une nouvelle atélectasie, qui sera visible à la radiographie pulmonaire. On devrait administrer les analgésiques appropriés, faire un examen pour trouver les causes sous-jacentes de la douleur, recommander la mobilité aux patients et les envoyer en physiothérapie.

\section{Insuffisance cardiaque congestive}

Selon de vastes études rétrospectives, environ $13 \%$ des réhospitalisations après un pontage aortocoronarien sont le résultat d'une insuffisance cardiaque, avec un taux supérieur observé chez les patients présentant une faible fraction d'éjection avant l'intervention chirurgicale ${ }^{18,19}$. Les atteintes myocardiques préopératoires irréversibles, les lésions myocardiques peropératoires, les lésions résiduelles aux artères coronaires menant à une ischémie continue, le dysfonctionnement diastolique ou l'hibernation myocardique après un pontage aortocoronarien sont des causes possibles. Les patients devraient être dirigés vers des services de cardiologie pour une prise en charge médicale selon la mise à jour complète de 2017 de la Société canadienne de cardiologie portant sur la prise en charge de l'insuffisance cardiaque ${ }^{20}$ - le titrage diurétique est la principale méthode de prise en charge.

\section{Pneumonie}

Une importante étude de cohorte prospective menée dans 10 centres affiliés aux National Institutes of Health et aux Instituts de recherche en santé du Canada a démontré une incidence de pneumonie de $2,4 \%$ après une chirurgie cardiaque ${ }^{21} ; 86 \%$ des cas se manifestaient dans les 30 jours suivant l'intervention, et seulement un tiers se produisait après le congé. La pneumonie postopératoire est habituellement due à l'utilisation d'un ventilateur, mais les patients sédentaires qui ne dégagent pas de façon adéquate leurs sécrétions pulmonaires risquent de contracter une pneumonie dans la communauté après leur congé de l'hôpital. Si une pneumonie se manifeste peu après le congé, il est important de prendre en considération les traitements empiriques qui offrent aussi une protection contre les micro-organismes nosocomiaux.

\section{Embolie pulmonaire}

L'embolie pulmonaire est peu fréquente après une chirurgie cardiaque en raison de l'administration systématique d'une thromboprophylaxie rigoureuse, du double traitement antiplaquettaire et de la mobilisation postopératoire précoce à l'hôpital. La poursuite de la mobilisation physique à domicile est aussi une méthode préventive. L'embolie pulmonaire survient habituellement de façon soudaine et entraîne une hypoxie importante. Chez les patients présentant une dyspnée grave, les médecins devraient examiner les causes les plus fréquentes de celle-ci tout en soupçonnant fortement une embolie pulmonaire.

\section{Quels problèmes cognitifs ou neurologiques les patients pourraient-ils présenter après l'intervention chirurgicale?}

Les complications neurologiques découlant d'une chirurgie cardiaque sont généralement divisées en déficits de type 1 et de type 2 . Les déficits de type 1 - accident vasculaire cérébral ou accident ischémique transitoire associé à une hypoperfusion périopératoire, à une embolisation ou à une hémorragie $e^{22,23}-$ se produisent chez environ $2 \%$ des patients subissant une chirurgie cardiaque $^{24}$, souvent tôt dans la période périopératoire. Ces événements sont pris en charge selon les lignes directrices standards pour la prise en charge d'accidents vasculaires cérébraux ${ }^{25}$. Les déficits de type 2 comprennent des complications neurocognitives plus particulières comme les dysfonctions cognitives postopératoires, le délirium et les troubles de l'humeur.

La dysfonction cognitive postopératoire est généralement décrite comme l'affaiblissement d'un domaine cognitif après une 
intervention chirurgicale, en l'absence de délirium et de démence ${ }^{26}$. Elle survient chez $50 \%-70 \%$ des patients au cours de la première semaine suivant l'opération. La plupart des patients se rétablissent, mais certains continuent à présenter une dysfonction cognitive (20\%-40\% des cas après $\left.1 \mathrm{an}^{27}\right)$ et d'autres déclinent même davantage. On ne sait pas encore si ce déclin peut être causé ou exacerbé par un déclin cognitif sous-jacent non diagnostiqué déjà présent chez les aînés avant l'intervention chirurgicale. Un déclin cognitif comparable a été démontré chez des patients dont la coronaropathie est prise en charge médicalement ${ }^{28}$.

Le délirium peut se produire chez un grand nombre de patients, jusqu'à $73 \%$ d'entre eux, après une chirurgie cardiaque $^{29}$, et l'anxiété, la dépression ou la psychose peuvent se manifester chez un tiers des patients après l'intervention ${ }^{30}$. La physiopathologie des complications de type 2 n'est pas entièrement comprise, mais les micro-emboles, l'hypoperfusion, l'hyperthermie cérébrale, les gaz anesthésiants et le syndrome de réponse inflammatoire systémique sont des mécanismes potentiels reconnus.

Les facteurs de risque pour la dysfonction de type 2 comprennent le grand âge, la présence d'une dysfonction cognitive préopératoire et des troubles psychiatriques ou de l'humeur prémorbides $^{31}$. Les déficits de types 2 les plus évidents sont souvent traités à l'hôpital, mais des déficiences cognitives subtiles peuvent n'être décelées que plus tard par les personnes qui prennent soin du patient à domicile. Les professionnels en soins primaires devraient être sensibilisés aux effets importants d'une déficience cognitive persistante sur le fonctionnement social, l'autonomie et le manque de reconnaissance des changements de la santé physique. À notre connaissance, il n'existe pas de données probantes ni de lignes directrices sur la manière de prendre en charge de subtiles déficiences neurocognitives après une chirurgie cardiaque; nous suggérons donc aux professionnels en soins primaires d'adhérer à leur pratique habituelle pour la prise en charge et le suivi des patients touchés par ces enjeux.

\section{Quelles autres complications pourraient survenir après une chirurgie cardiaque?}

\section{Complications associées au site opératoire}

Les médecins de famille qui voient des patients dans les 30 jours suivant un pontage aortocoronarien devraient procéder à un examen systématique de tous les sites opératoires, comme montré à l'annexe 1 (accessible en anglais au www.cmaj.ca/lookup/ doi/10.1503/cmaj.191108/tab-related-content), au cours des rendez-vous de suivi afin de vérifier la présence d'infections superficielles ou profondes ou de repérer une possible désunion du médiastin («clic » sternal non sensible et palpable). La détection précoce et l'intervention du chirurgien cardiaque ont le potentiel de prévenir des complications très graves, les lésions sternales pouvant augmenter la morbidité et la mortalité de façon importante, soit jusqu'à $40 \% 32$. Les caractéristiques des plaies chirurgicales et les considérations importantes en lien avec celles-ci sont présentées en détail à l'annexe 2, tableau 1 (accessible en anglais au www.cmaj. ca/lookup/doi/10.1503/cmaj.191108/tab-related-content).

\section{Insuffisance rénale}

Jusqu'à $30 \%$ des patients développent une insuffisance rénale aiguë de gravité variable après une chirurgie cardiaque ${ }^{33}$. Ce problème est généralement le résultat d'une hypoperfusion rénale ou de complications emboliques périopératoires. Environ $1 \%$ des patients ont besoin de dialyse après une chirurgie cardiaque ${ }^{33}$. La plupart des patients présentant une insuffisance rénale aiguë postopératoire ont des facteurs de risque préopératoires (p. ex., âge avancé, athérosclérose, diabète, insuffisance rénale ou cardiaque préopératoire) qui les prédisposent à une insuffisance rénale aiguë. Cette dernière est souvent transitoire, mais les patients qui présentent une dysfonction rénale préexistante peuvent présenter une dégradation à long terme de leur insuffisance rénale, qui nécessitera un suivi et une prise en charge continus et attentifs.

\section{Les défis du contrôle du diabète}

Les Lignes directrices de pratique clinique 2018 de Diabète Canada soulignent qu'un mauvais contrôle glycémique périopératoire est associé à une augmentation des complications après la chirurgie cardiaque, dont l'infection de la plaie sternale, le délirium, l'insuffisance rénale, la dysfonction respiratoire et la prolongation de l'hospitalisation ${ }^{34}$. Afin d'éviter ces complications, les médecins qui soignent périopératoirement les patients diabétiques ajustent souvent le traitement du diabète afin d'assurer un contrôle glycémique plus serré. Le suivi postopératoire avec un professionnel en soins primaires est crucial pour évaluer le contrôle et le traitement continu du diabète, ciblant préférablement un taux d'hémoglobine glyquée d'au plus $7 \%$ selon les Lignes directrices de pratique clinique de Diabète Canada ${ }^{35}$.

\section{Saignement gastro-intestinal}

Le saignement gastro-intestinal est une complication peu fréquente mais importante chez les patients qui ont subi une chirurgie cardiaque, surtout s'ils présentaient une pathologie gastro-intestinale préexistante. Les traumatismes peropératoires encourus lors de l'échocardiographie transœsophagienne, les ulcères de stress et la prise d'anti-inflammatoires non stéroïdiens prescrits en milieu hospitalier contribuent aux saignements gastro-intestinaux. Les patients à qui on administre en plus une anticoagulothérapie risquent de présenter des saignements gastro-intestinaux très importants. En présence d'un saignement gastro-intestinal, la décision de poursuivre ou non l'anticoagulothérapie est idéalement prise en concertation avec le chirurgien cardiaque. La prise en charge devrait suivre les pratiques exemplaires; une hémorragie devrait toujours entraîner la cessation de l'anticoagulothérapie, l'orientation vers un service des urgences et la prise de contact avec le chirurgien cardiaque de garde.

\section{Comment l'anticoagulothérapie devrait-elle être prise en charge après une chirurgie cardiaque?}

\section{L'anticoagulothérapie pour la fibrillation auriculaire}

La fibrillation auriculaire se manifeste chez $30 \%$ des patients après un pontage aortocoronarien isolé ${ }^{36}$. Les lignes directrices de 2020 de la Société canadienne de cardiologie sur la prise en charge de la 
fibrillation auriculaire recommandent que le traitement pour la fibrillation auriculaire après une chirurgie cardiaque soit personnalisé selon le contrôle du taux ou du rythme ${ }^{36}$. Les patients peuvent donc obtenir leur congé avec une prescription de bêtabloquants, d'amiodarone ou des deux, qui peut être adaptée par un professionnel en soins primaires au besoin. Afin de diminuer leur risque d'accident vasculaire cérébral, les patients peuvent aussi recevoir une prescription d'anticoagulants, plus précisément de warfarine, au moment de leur congé si leur fibrillation auriculaire persiste durant plus de 48 heures ${ }^{37}$. La fibrillation auriculaire secondaire à un pontage aortocoronarien est spontanément résolutive, et presque tous les patients retrouvent un rythme sinusal normal dans les 6-12 semaines après la chirurgie cardiaque ${ }^{36,38,39}$. Par conséquent, les professionnels en soins primaires qui suivent les patients devraient toujours réévaluer la pertinence de l'anticoagulothérapie 3 mois après l'intervention ${ }^{36,39}$. L'arrêt de ce traitement devrait alors être envisagé si le patient ne présente plus d'arythmie (rythme sinusal normal, aucune palpitation) et que ses seules manifestations de fibrillation auriculaire sont survenues après la chirurgie cardiaque $\mathrm{e}^{36,39}$.

\section{Comment l'hypertension devrait-elle être prise en charge après un pontage aortocoronarien?}

Plusieurs patients présentant une hypertension préopératoire voient leur dose d'antihypertenseurs réduite au moment du congé $^{16}$. Cette diminution est due en partie au risque de syndrome de réponse inflammatoire systémique associé à la dérivation cardiopulmonaire et aux changements volémiques après l'intervention $^{40}$. Le processus inflammatoire se résorbe avec le temps (en quelques jours), mais peut avoir des effets persistant plusieurs semaines après la chirurgie. Cet état de fait, combiné avec l'anémie, la diminution transitoire de la fonction cardiaque et la diurèse continue après l'intervention, signifie que la tension artérielle du patient peut être momentanément plus basse qu'avant l'intervention. L'hypertension peut devenir manifeste à mesure que le rétablissement progresse et peut nécessiter l'ajustement des antihypertenseurs au cours des mois suivants, selon les lignes directrices canadiennes de 2020 sur l'hypertension.

\section{Comment les patients devraient-ils aborder la pratique d'activités après l'intervention chirurgicale?}

\section{Réadaptation}

Les patients sont généralement dirigés vers un établissement de réadaptation cardiaque par leur centre chirurgical pour commencer un traitement 6-12 semaines après l'intervention. Certaines provinces canadiennes offrent des programmes de réadaptation par des physiothérapeutes communautaires ${ }^{42}$. Un examen exhaustif a montré que la réadaptation cardiaque est associée à une diminution de la mortalité après l'intervention chirurgicale ${ }^{43}$. Si les programmes recommandés ne sont pas disponibles, on encourage les patients à augmenter leurs activités

Considérations en matière de suivi de pontage aortocoronarien

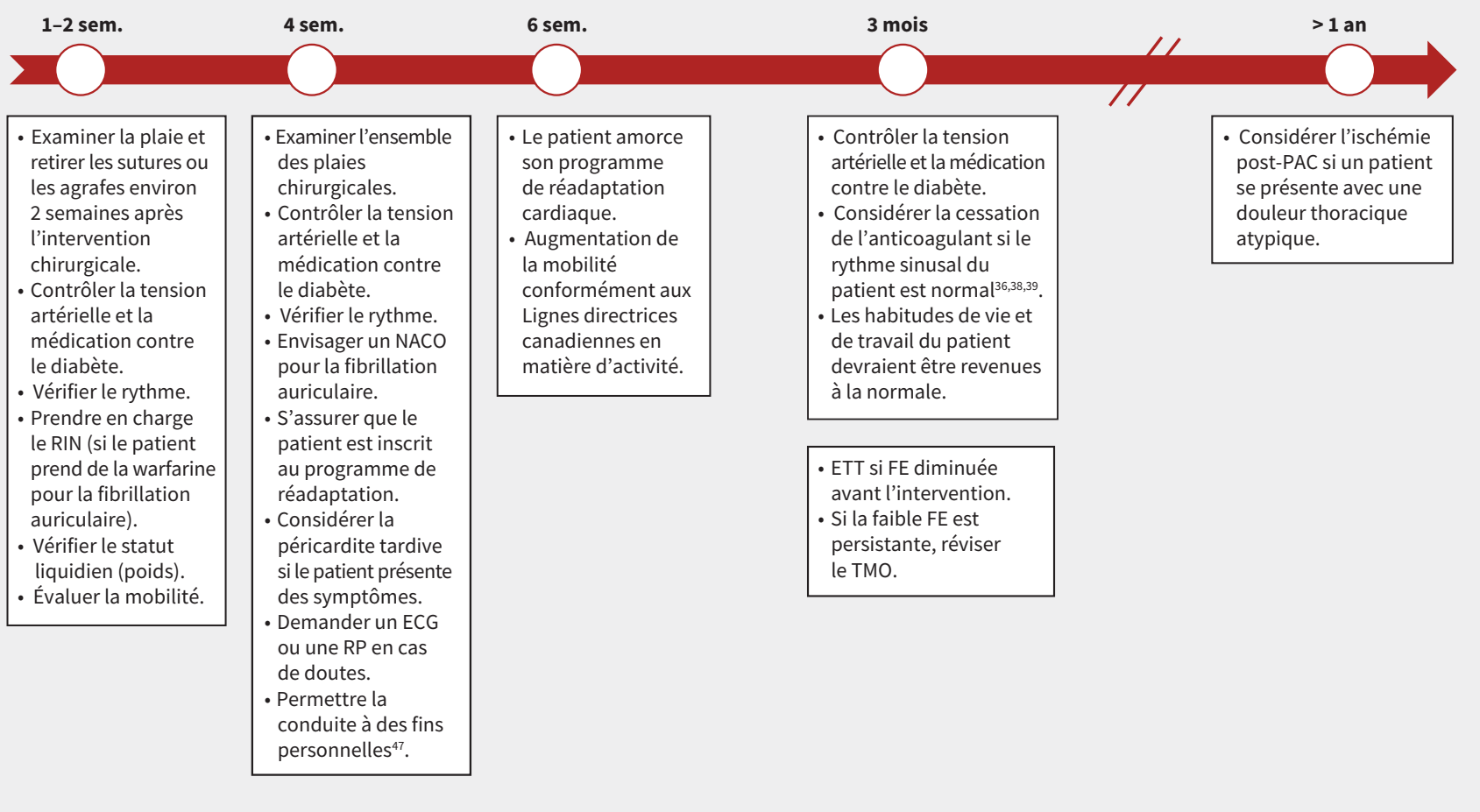

Figure 1 : Chronologie du suivi postopératoire suggéré avec les fournisseurs de soins primaires. Remarque : ECG = électrocardiogramme, ETT = échocardiographie transthoracique, $\mathrm{FE}=$ fraction d'éjection, $\mathrm{NACO}=$ nouvel anticoagulant oral, $\mathrm{PAC}=$ pontage aortocoronarien, RIN = rapport international normalisé, $\mathrm{RP}=$ radiographie pulmonaire, $\mathrm{TMO}=$ traitement médical optimal. 
graduellement, selon leur tolérance, pour atteindre les recommandations de la Société canadienne de physiologie de l'exercice, soit 150 minutes d'exercices aérobiques d'intensité modérée à vigoureuse par semaine ${ }^{44}$. En pratique, ceci est aussi simple qu'augmenter la distance et la vitesse de marche quotidienne jusqu'à avoir atteint ce but. Comme nous l'avons décrit auparavant, il est important que les patients ne deviennent pas sédentaires à domicile après avoir obtenu leur congé.

\section{Précautions sternales}

La plupart des centres chirurgicaux vont offrir des recommandations aux patients concernant la manière d'éviter les blessures sternales. Les conseils classiques comprennent d'éviter de tirer, de pousser ou de soulever des charges de plus de 10 livres (5 kg), d'éviter les mises en charge des bras et d'éviter les rotations du haut du corps durant les 6-12 semaines suivant l'intervention chirurgicale ${ }^{17,45}$. De nouvelles stratégies ont été élaborées, dont la technique Keep Your Move in the Tube ( Gardez vos mouvements dans le cylindre ») qui encourage la mobilité après une chirurgie cardiaque en s'éloignant de la mise en charge et des contraintes de temps et en se concentrant sur des principes de kinésiologie ${ }^{46}$. Cette dernière technique recommande aux patients de laisser le haut de leurs bras près de leur corps (comme s'ils étaient dans un cylindre) afin d'éviter le stress excessif sur le sternum pendant les mouvements de mise en charge. La reprise des activités normales d'un patient se fait à son propre rythme en s'assurant de l'absence de douleur à la poitrine ou au point d'incision ${ }^{46}$.

\section{Conduite}

Selon le sommaire de 2003 de la Société canadienne de cardiologie sur l'évaluation de l'aptitude à conduire après une chirurgie cardiaque, il est permis de conduire 4 semaines après un pontage aortocoronarien ${ }^{47}$.

\section{Conclusion}

Les professionnels des soins primaires jouent un rôle vital dans les soins qui suivent le congé de patients ayant subi un pontage aortocoronarien. Ces soins comprennent entre autres le suivi des patients pour d'importantes complications éventuelles pendant leur rétablissement, la révision et l'optimisation de la médication, l'orientation appropriée du patient si des complications surgissent et la supervision d'une reprise sécuritaire des activités et du fonctionnement. Un résumé des considérations de suivi sous la forme d'échéancier (figure 1) est fourni comme guide de référence rapide.

\section{Références}

1. Cardiac Care Quality Indicators Report. Ottawa: Canadian Institute for Health Information; 2017.

2. Arora RC, Manji RA, Singal RK, et al. Outcomes of octogenarians discharged from the hospital after prolonged intensive care unit length of stay after cardiac surgery. J Thorac Cardiovasc Surg 2017;154:1668-78.e2.

3. Kirklin JW, Kouchoukos NT. Kirklin/Barratt-Boyes cardiac surgery: morphology, diagnostic criteria, natural history, techniques, results, and indications. 4th ed. Philadelphia: Elsevier/Saunders; 2013.

4. Imazio M, Hoit BD. Post-cardiac injury syndromes. An emerging cause of pericardial diseases. Int J Cardiol 2013;168:648-52.
5. Imazio M, Brucato A, Rovere ME, et al. Contemporary features, risk factors, and prognosis of the post-pericardiotomy syndrome. Am J Cardiol 2011;108:1183-7.

6. Adler Y, Charron P, Imazio M, et al.; ESC Scientific Document Group. 2015 ESC guidelines for the diagnosis and management of pericardial diseases: the task force for the diagnosis and management of pericardial diseases of the European Society of Cardiology (ESC) endorsed by: the European Association for CardioThoracic Surgery (EACTS). Eur Heart J 2015;36:2921-64.

7. Imazio M, Brucato A, Cemin R, et al.; ICAP Investigators. A randomized trial of colchicine for acute pericarditis. N Engl J Med 2013;369:1522-8.

8. Hardesty RL, Thompson M, Lerberg DB, et al. Delayed postoperative cardiac tamponade: diagnosis and management. Ann Thorac Surg 1978;26:155-64.

9. Jensen JK, Poulsen SH, Mølgaard H. Cardiac tamponade: a clinical challenge. e-Journal of Cardiology Practice 2017;15.

10. Khan NK, Järvelä KM, Loisa EL, et al. Incidence, presentation and risk factors of late postoperative pericardial effusions requiring invasive treatment after cardiac surgery. Interact Cardiovasc Thorac Surg 2017;24:835-40.

11. Serhiyenko VA, Serhiyenko AA. Cardiac autonomic neuropathy: risk factors, diagnosis and treatment. World J Diabetes 2018;9:1-24.

12. Belley-Cote EP, Lamy A, Devereaux PJ, et al. Definitions of post-coronary artery bypass grafting myocardial infarction: variations in incidence and prognostic significance. Eur J Cardiothorac Surg 2020;57:168-75.

13. Morice M-C, Serruys PW, Kappetein AP, et al. Five-year outcomes in patients with left main disease treated with either percutaneous coronary intervention or coronary artery bypass grafting in the synergy between percutaneous coronary intervention with taxus and cardiac surgery trial. Circulation 2014;129:2388-94.

14. Alahdab F, Jazayerli ML, Alhalabi O, et al. Aspirin dosage for the prevention of graft occlusion in people undergoing coronary surgery. Cochrane Database Syst Rev 2016;(3):CD012113.

15. Tanguay J-F, Bell AD, Ackman ML, et al.; Canadian Cardiovascular Society. Focused 2012 update of the Canadian Cardiovascular Society guidelines for the use of antiplatelet therapy. Can J Cardiol 2013;29:1334-45.

16. Kulik A, Ruel M, Jneid H, et al.; American Heart Association Council on Cardiovascular Surgery and Anesthesia. Secondary prevention after coronary artery bypass graft surgery: a scientific statement from the American Heart Association. Circulation 2015;131:927-64.

17. Conte JV, Baumgartner WA, Owens SGT, et al. The Johns Hopkins manual of cardiac surgical care. 2nd ed. Milton (ON): Elsevier Canada; 2008.

18. Hannan EL, Zhong Y, Lahey SJ, et al. 30-day readmissions after coronary artery bypass graft surgery in New York State. JACC Cardiovasc Interv 2011;4:569-76.

19. Moreyra AE, Deng Y, Wilson AC, et al. Incidence and trends of heart failure admissions after coronary artery bypass grafting surgery. Eur J Heart Fail 2013; 15:46-53.

20. Ezekowitz JA, O'Meara E, McDonald MA, et al. 2017 comprehensive update of the Canadian Cardiovascular Society guidelines for the management of heart failure. Can J Cardiol 2017;33:1342-433.

21. Ailawadi G, Chang HL, O'Gara PT, et al. Pneumonia after cardiac surgery: experience of the National Institutes of Health/Canadian Institutes of Health Research Cardiothoracic Surgical Trials Network. J Thorac Cardiovasc Surg 2017;153:1384-91.e3.

22. Sylivris S, Levi C, Matalanis G, et al. Pattern and significance of cerebral microemboli during coronary artery bypass grafting. Ann Thorac Surg 1998;66: 1674-8.

23. Newman MF, Kramer D, Croughwell ND, et al. Differential age effects of mean arterial pressure and rewarming on cognitive dysfunction after cardiac surgery. Anesth Analg 1995;81:236-42.

24. Whitlock R, Healey JS, Connolly SJ, et al. Predictors of early and late stroke following cardiac surgery. CMAJ 2014;186:905-11.

25. Powers WJ, Rabinstein AA, Ackerson T, et al. 2018 guidelines for the early management of patients with acute ischemic stroke: a guideline for healthcare professionals from the American Heart Association/American Stroke Association. Stroke 2018;49:e46-110.

26. Tsai TL, Sands LP, Leung JM. An update on postoperative cognitive dysfunction. Adv Anesth 2010;28:269-84. 
27. Newman MF, Mathew JP, Grocott HP, et al. Central nervous system injury associated with cardiac surgery. Lancet 2006;368:694-703.

28. McKhann GM, Grega MA, Borowicz LM Jr, et al. Is there cognitive decline 1 year after CABG? Comparison with surgical and nonsurgical controls. Neurology 2005;65:991-9.

29. Arora RC, Djaiani G, Rudolph JL. Detection, prevention, and management of delirium in the critically ill cardiac patient and patients who undergo cardiac procedures. Can J Cardiol 2017;33:80-7.

30. Horne D, Kehler S, Kaoukis G, et al. Depression before and after cardiac surgery: Do all patients respond the same? J Thorac Cardiovasc Surg 2013;145:1400-6.

31. Indja B, Seco M, Seamark R, et al. Neurocognitive and psychiatric issues post cardiac surgery. Heart Lung Circ 2017;26:779-85.

32. Shih C-C, Shih C-M, Su Y-Y, et al. Potential risk of sternal wires. Eur J CardiothoraC Surg 2004;25:812-8.

33. Rosner MH. Acute kidney injury associated with cardiac surgery. Redo Card Surg Adults 2012;9781461413:37-52.

34. Diabetes Canada Clinical Practice Guidelines Expert Committee; Malcolm J, Halperin I, Miller DB, et al. In-hospital management of diabetes. Can J Diabetes 2018;42(Suppl 1):S115-23.

35. Diabetes Canada Clinical Practice Guidelines Expert Committee; Imran SA, Agarwal G, Bajaj HS, et al. Targets for glycemic control. Can J Diabetes 2018;42 (Suppl 1):S42-6.

36. Andrade JG, Aguilar M, Atzema C, et al. The 2020 Canadian Cardiovascular Society/Canadian Heart Rhythm Society comprehensive guidelines for the management of atrial fibrillation. Can J Cardiol 2020;36:1847-948.

37. January CT, Wann LS, Alpert JS, et al.; American College of Cardiology/American Heart Association Task Force on Practice Guidelines. 2014 AHA/ACC/HRS guideline for the management of patients with atrial fibrillation: a report of the American College of Cardiology/American Heart Association Task Force on Practice Guidelines and the Heart Rhythm Society. J Am Coll Cardiol 2014;64:e1-76.

38. Bessissow A, Khan J, Devereaux PJ, et al. Postoperative atrial fibrillation in noncardiac and cardiac surgery: an overview. J Thromb Haemost 2015;13(Suppl 1): S304-12.

39. Mitchell LB; CCS Atrial Fibrillation Guidelines Committee. Canadian Cardiovascular Society atrial fibrillation guidelines 2010: prevention and treatment of atrial fibrillation following cardiac surgery. Can J Cardiol 2011;27:91-7.

40. Kerbaul F, Collart F, Giorgi R, et al. Role of endogenous adenosine as a predictive marker of vasoplegia during cardiopulmonary bypass and postoperative severe systemic inflammatory response. Crit Care Med 2006;34:640-5.

41. Rabi DM, McBrien KA, Sapir-Pichhadze R, et al. Hypertension Canada's 2020 comprehensive guidelines for the prevention, diagnosis, risk assessment, and treatment of hypertension in adults and children. Can J Cardiol 2020;36: 596-624.
42. Tran M, Pesah E, Turk-Adawi K, et al. Cardiac rehabilitation availability and delivery in Canada: How does it compare with other high-income countries? Can J Cardiol 2018;34(Suppl 2):S252-62.

43. Price KJ, Gordon BA, Bird SR, et al. A review of guidelines for cardiac rehabilitation exercise programmes: Is there an international consensus? Eur J Prev Cardiol 2016;23:1715-33.

44. Tremblay MS, Warburton DER, Janssen I, et al. New Canadian physical activity guidelines. Appl Physiol Nutr Metab 2011;36:36-46.

45. Cahalin LP, Lapier TK, Shaw DK. Sternal precautions: Is it time for change? Precautions versus restrictions - A review of literature and recommendations for revision. Cardiopulm Phys Ther J 2011;22:5-15.

46. Adams J, Lotshaw A, Exum E, et al. An alternative approach to prescribing sternal precautions after median sternotomy, "Keep your move in the tube". Proc Bayl Univ Med Cent 2016;29:97-100.

47. Simpson C, Ross D, Dorian P, et al. CCS Consensus Conference 2003: Assessment of the cardiac patient for fitness to drive and fly - Executive summary. Can J Cardiol 2004;20:1313-20.

\section{Intérêts concurrents : Aucun déclaré}

Cet article a été révisé par des pairs.

Affiliations : Faculté de médecine (de Waard, Fagan, Horne), Université Dalhousie; Division de la chirurgie cardiaque (de Waard, Fagan, Horne), Hôpital Queen Elizabeth II, Halifax, N.-É.; Hôpital Bethesda (Minnaar), Steinbach, Man.

Collaborateurs : Dominique de Waard, Andrew Fagan et David Horne ont contribué à l'élaboration et à la conception du travail. Tous les auteurs ont contribué à l'ébauche du manuscrit, en ont révisé de façon critique le contenu intellectuel important, ont donné leur approbation finale pour la version destinée à être publiée, et assument l'entière responsabilité de tous les aspects du travail.

Propriété intellectuelle du contenu : Il s'agit d'un article en libre accès distribué conformément aux modalités de la licence Creative Commons Attribution (CC BY-NC-ND 4.0), qui permet l'utilisation, la diffusion et la reproduction dans tout médium à la condition que la publication originale soit adéquatement citée, que l'utilisation se fasse à des fins non commerciales (c.-à-d., recherche ou éducation) et qu'aucune modification ni adaptation n'y soit apportée. Voir : https:// creativecommons.org/licenses/by-nc-nd/4.0/deed.fr.

Correspondance : David Horne, David.Horne@iwk.nshealth.ca 